\title{
Wireless Energy Harvesting in Two-Way Network Coded Cooperative Communications: A Stochastic Approach for Large Scale Networks
}

\author{
Prodromos-Vasileios Mekikis, Student Member, IEEE, Aris S. Lalos, Member, IEEE, \\ Angelos Antonopoulos, Member, IEEE, Luis Alonso, Senior Member, IEEE, and Christos Verikoukis, Senior \\ Member, IEEE
}

\begin{abstract}
In this letter, we study the performance of Network Coding (NC)-aided cooperative communications in large scale networks, where the relays are able to harvest energy emitted by wireless transmissions. In particular, we derive theoretical expressions for key network performance metrics, i.e., the probability of successful data exchange and the network lifetime gain. The proposed analytical expressions are verified via extensive Monte Carlo simulations, demonstrating the potential benefits of the energy harvested by the wireless transmissions.
\end{abstract}

Index Terms-Bidirectional Cooperative Networks, Network Coding, Energy Harvesting, Stochastic Geometry

\section{INTRODUCTION}

$\mathbf{T}$ HE incorporation of Network Coding (NC) [1]-[4] in bidirectional cooperative networks leads to significant capacity improvement by enabling the relays to process the incoming data (using the XOR function) before forwarding them to the respective destinations. However, the relay nodes are often powered by limited capacity batteries. Therefore, their lifetime is a crucial performance metric [5] that should be also considered.

The limited lifetime of the nodes in Wireless Sensor Networks (WSNs) motivated researchers and system designers to study the application of Energy Harvesting (EH), allowing the increase of the network lifetime by collecting energy from various sources (e.g., solar, wind, etc.). However, in cases where the aforementioned sources are not available, it is still possible to harvest energy from the electromagnetic radiation (EMR) of the transmissions in the network [6]. Furthermore, as technology evolves, the number of wireless devices constantly increases, creating large scale networks. The nodes of these networks interfere to each other while, at the same time, contribute to the EMR energy that can be harvested.

To that end, Stochastic Geometry [7] can be applied to study both the network performance and the lifetime gains offered by EMR EH. To the best of our knowledge, only two works study the impact of EMR energy harvesting in large scale networks [8], [9]. Both works consider direct communication scenarios and give useful insights on the potential benefits of

P.-V. Mekikis, A. S. Lalos and L. Alonso are with the Department of Signal Theory and Communications (TSC), Polytechnic University of Catalonia (UPC), Spain, (e-mail: \{vmekikis, aristeidis.lalos, luisg \}@tsc.upc.edu)

A. Antonopoulos and $\mathrm{Ch}$. Verikoukis are with the Telecommunications Technological Centre of Catalonia (CTTC), Spain, (e-mail: \{aantonopoulos, cveri\}@cttc.es). the EMR EH technology. However, they do not characterize the performance of general cooperative networks. In addition, the analysis is made under assumptions that are either not applicable in WSNs or based on specific physical layer configurations. More precisely, [8] assumes power adaptation capabilities for the nodes and, in [9], closed-form expressions for the network metrics are provided only for a particular value of the path loss exponent.

In this paper, we consider a large scale two-way NCaided cooperative network, where the relays have EMR EH capabilities, in order to be able to assist the sources to exchange their data for longer time periods. Our contribution can be summarized as follows: i) we provide a closedform expression for the lower bound of the probability of successful data exchange (which is an end-to-end Quality of Service (QoS) metric) for every path loss exponent, and ii) we theoretically express the lifetime gain thanks to the usage of EMR harvesting at the relays. The provided closed-form expressions can be applied in QoS optimization problems with energy lifetime constrains (or vice versa) [10], for bidirectional cooperative networks.

The rest of the paper is organized as follows. Section II describes the system model. Section III presents the theoretical analysis for the successful message exchange and the network lifetime. Section IV includes the model validation and the experimental results. Finally, Section V concludes the paper.

\section{SySTEM MODEL}

We consider a large scale network consisting of two sets of sources $S_{1}$ and $S_{2}$ in saturated conditions that exchange messages with the assistance of relays $R$. All nodes are assumed to be moving on the same Euclidean plane and they are represented by three independent homogeneous Poisson Point Processes (PPPs), a sensible approach for wireless networks [7] [8]. The sources $S_{1}$ are described by the PPP $\Phi_{S_{1}}=$ $\left\{x_{1}, \ldots, x_{i}\right\}$, where $x_{i}, \forall i \in \mathbb{N}$, denotes the location of the $S_{1}$ source $s_{1 i} . \Phi_{S_{1}}$ has an intensity $\lambda_{1}$, which corresponds to the average number of points per area unit. Accordingly, the PPP $\Phi_{S_{2}}=\left\{y_{1}, \ldots, y_{j}\right\}$ with intensity $\lambda_{2}$ represents the location $y_{j}, \forall j \in \mathbb{N}$, of the $S_{2}$ source $s_{2 j}$ and $\Phi_{R}=\left\{z_{1}, \ldots, z_{k}\right\}$, $\forall k \in \mathbb{N}$, with intensity $\lambda_{R}$, the location $z_{k}$ of the relay $r_{k}$.

All nodes are assumed to be equipped with single-inputsingle-output transceivers and the sources $\left(S_{1}, S_{2}\right)$ have identical capabilities. Moreover, all nodes are powered by a battery 


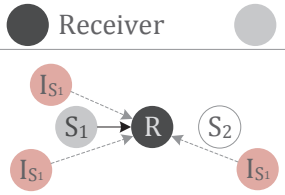

(a)

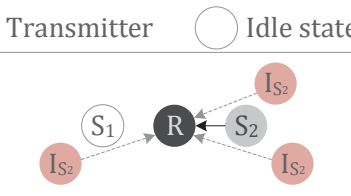

(b)
Interferer

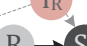

$\mathrm{R} \rightarrow \mathrm{S}_{2}$

(c)
Fig. 1. Communication phases: a) Slot $1\left(S_{1} \rightarrow R\right)$, b) Slot $2\left(S_{2} \rightarrow R\right)$, c) Slot $3\left(R \stackrel{N C}{\longrightarrow} S_{1}, S_{2}\right)$

with initial energy level $L_{I}$. In addition, the relays are capable of EMR EH using a separate EMR Harvesting System (EHS) with a rectifying antenna (rectenna), which is a special type of antenna that is used to convert RF energy into direct current electricity with efficiency $\epsilon$ [11]. A relay is able to harvest the EMR energy that emits from the transmissions of the sources, of the other relays and of its own transmissions.

The time is divided into $m$ "communication periods" $T_{m}$, where $m \in \mathbb{N}$. Each communication period consists of three time slots of duration $t_{s}$. In the first time slot, each source $s_{1 i}$ is transmitting its message to the closest relay $r_{k}$, as depicted in Fig. 1(a). The rest of the sources are considered as interference for the relay that is trying to decode the message of its associated $S_{1}$ source. At the same time, the EMR received by the relay due to the transmissions of all $S_{1}$ sources is harvested by the EHS. In the second time slot, the $S_{1}$ sources are idle and each $S_{2}$ source $s_{2 j}$ is transmitting its message to the closest relay, as depicted in Fig. 1(b). Again, the rest of the $S_{2}$ sources are considered as interference at the relay, which at the same time harvests the incoming energy. Finally, in the third time slot, illustrated in Fig. 1(c), each relay that has received and successfully decoded the messages from its closest $S_{1}$ and $S_{2}$ sources is helping to the exchange of the messages by broadcasting the NC message to the two sources.

For our analysis, without loss of generality, we assume that the respective receiving node in each slot is located at the origin (Slyvnyak's theorem [7]). The received power at a node located in a distance $d$ from the transmitting node is $P_{t} h d^{-\alpha}$, where $P_{t}$ is the transmission power of the nodes, $\alpha>2$ is the path loss exponent and $h$ is the square of the amplitude fading coefficient (i.e., the power fading coefficient) that is associated with the channel between the nodes. We also assume that the fading coefficients are independent and identically distributed (i.i.d.). Moreover, the amplitude fading $\sqrt{h}$ is Rayleigh with a scale parameter $\sigma=1$, hence $h$ is exponentially distributed with mean value $\mu=1$. A message is considered to be successfully decoded at a receiver when its signal-to-noise-plus-interference ratio (SINR) from its nearest transmitter is higher than a threshold $\gamma$; otherwise the message is dropped. The SINR of a mobile node located at the origin at a distance $d$ from its associated transmitter is defined as

$$
\mathrm{SINR}=\frac{P_{t} h d^{-\alpha}}{I_{d}+N},
$$

where the interference is defined as $I_{d}=\sum_{x \in \Phi} P_{t} h_{x} x^{-\alpha}$ and $N$ is the additive white Gaussian noise power that is modeled as a constant zero mean Gaussian Random Variable (RV).

\section{QOS AND LIFETIME ANALYSIS}

In this section, we provide the theoretical derivations of two important metrics for the network performance: i) the probability of successful data exchange between two nodes, and ii) the lifetime gain thanks to the EMR EH.

\section{A. Successful message exchange probability}

The end-to-end QoS metric of our interest is the probability of successful message exchange, denoted as $p_{e x}$. This metric is written as

$$
p_{\text {ex }}=p_{\text {act }} p_{n c}=\left(p_{s_{1} \rightarrow r} p_{s_{2} \rightarrow r}\right)\left(p_{r \rightarrow s_{1}} p_{r \rightarrow s_{2}}\right),
$$

where $p_{t x \rightarrow r x}$, with $t x, r x=\left\{s_{1}, s_{2}, r\right\}$, is the probability that the receiver $r x$ will decode successfully the message from transmitter $t x, p_{a c t}=p_{s_{1} \rightarrow r} p_{s_{2} \rightarrow r}$ is the probability that the relay is active during the third slot (i.e., the relay has successfully received the messages in the first two slots), and $p_{n c}=p_{r \rightarrow s_{1}} p_{r \rightarrow s_{2}}$ is the probability that the sources have successfully received the network coded message from the relay in the third slot.

Eq.(2) suggests that the probability of successful message exchange depends exclusively on the probability of successful decoding of each individual transmission. Thus, we will apply the analysis for the probability of successful decoding $p_{t x \rightarrow r x}$ at one random receiver node and, then, we will adjust accordingly the parameters for the nodes of type $S_{1}, S_{2}$ and $R$. To that end, the probability of successful decoding is defined as

$$
\begin{aligned}
& p_{t x \rightarrow r x}=\operatorname{Pr}(\operatorname{SINR}>\gamma)=\operatorname{Pr}\left(\frac{P_{t} h d^{-\alpha}}{I_{d}+N}>\gamma\right) \\
& \stackrel{(a)}{=} \int_{0}^{\infty} \int_{0}^{\infty} \operatorname{Pr}\left(h>\frac{t_{1}^{\alpha} \gamma\left(t_{2}+N\right)}{P_{t}}\right) f_{d}\left(t_{1}\right) f_{I_{d}}\left(t_{2}\right) \mathrm{d} t_{1} \mathrm{~d} t_{2} \\
& \stackrel{(b)}{=} \mathbb{E}_{d}\left\{\mathbb{E}_{I_{d}}\left\{\exp \left(-\frac{t_{1}^{\alpha}}{P_{t}} \gamma\left(t_{2}+N\right)\right)\right\}\right\} \\
& \stackrel{(c)}{=} \mathbb{E}_{d}\left\{\mathcal{L}_{I_{d}}\left(\frac{t_{1}^{\alpha}}{P_{t}} \gamma\right) \exp \left(-\frac{t_{1}^{\alpha}}{P_{t}} \gamma N\right)\right\},
\end{aligned}
$$

where Eq.(3a) follows from the independence of the RVs $d$ and $I_{d}$ with probability density functions (PDFs) $f_{d}$ and $f_{I_{d}}$ and Eq.(3b) follows from the cumulative distribution function (CDF) of an exponential RV with unit variance. Finally, Eq.(3c) follows from the definition of the Laplace transform. More specifically, by following the guidelines of [7, 5.1.7] and after some algebra, the Laplace transform of the PDF of $I_{d}$ caused at a receiver can be expressed as

$$
\mathcal{L}_{I_{d}}(s)=\exp \left(-\frac{2 \lambda \pi^{2}\left(P_{t} s\right)^{\frac{2}{\alpha}}}{\alpha \sin (2 \pi / \alpha)}\right) .
$$

By substituting Eq.(4) in Eq.(3c), we may write

$$
p_{t x \rightarrow r x}=\mathbb{E}_{d}\{\underbrace{\exp \left(-\frac{2 \lambda \pi^{2}\left(t_{1}^{\alpha} \gamma\right)^{\frac{2}{\alpha}}}{\alpha \sin (2 \pi / \alpha)}-\frac{t_{1}^{\alpha}}{P_{t}} \gamma N\right)}_{f\left(t_{1}\right)}\}
$$

Note that, $p_{t x \rightarrow r x}$ yields a closed-form expression only for the particular case of $\alpha=4$ [12]. To overcome this limitation, we employ Jensen's inequality in order to provide a lower bound for the probability of successful decoding for every $\alpha>2$ by using the expected value of the distance to the nearest node. Since the exponential function is convex, we can apply Jensen inequality on $\mathbb{E}_{d}\left\{f\left(t_{1}\right)\right\}$ given by

$$
f\left(\mathbb{E}_{d}\left\{t_{1}\right\}\right) \leq \mathbb{E}_{d}\left\{f\left(t_{1}\right)\right\} .
$$


The PDF of the distance to the nearest node from the origin is expressed as [7, 2.9.1]

$$
f_{d}\left(t_{1}\right)=2 \pi \lambda t_{1} \exp \left(-\lambda \pi t_{1}^{2}\right) .
$$

With regard to the distance to the nearest node, the expected value $\mathbb{E}_{d}\left\{t_{1}\right\}$ may be written as

$$
\mathbb{E}_{d}\left\{t_{1}\right\}=\int_{0}^{\infty} t_{1} f_{d}\left(t_{1}\right) \mathrm{d} t_{1} \stackrel{(a)}{=} 2 \pi \lambda \frac{\Gamma[3 / 2]}{2(\pi \lambda)^{3 / 2}} \stackrel{(b)}{=} \frac{1}{2 \sqrt{\lambda}},
$$

where Eq.(8a) follows from the definite integral formula provided in $[14,15.77(m=2, a=\pi \lambda)]$, the Gamma function is given by $\Gamma(z)=\int_{0}^{\infty} t^{z-1} e^{-t} \mathrm{~d} t$ and $\Gamma[3 / 2]=\frac{\sqrt{\pi}}{2}$. Hence, using Eq.(5), (6) and (8b), the probability of successful decoding is expressed as

$$
p_{t x \rightarrow r x} \geq \exp \left(\frac{-\pi^{2} \gamma^{2 / \alpha}}{2 \alpha \sin (2 \pi / \alpha)}\right) \exp \left(\frac{-\gamma N}{P_{t}\left(2 \sqrt{\lambda_{j}}\right)^{\alpha}}\right),
$$

where $\lambda_{j}$ is the intensity of the PPP on which we apply the probability, i.e., $j=\{1,2, R\}$. We notice that $\lambda_{j}$ is the only parameter that differentiates the probabilities of Eq.(2). By applying Eq.(9) to Eq.(2) using the appropriate $\lambda$ value for each probability, we can derive the probability of successful exchange $p_{e x}$ and the probability of active relay $p_{a c t}{ }^{1}$. Finally, the lower bound of the probability $p_{e x}$ is given by

$$
p_{e x}=\exp \left[\frac{-2 \pi^{2} \gamma^{\frac{2}{\alpha}}}{\alpha \sin (2 \pi / \alpha)}-\frac{\gamma N}{P_{t} 2^{\alpha}}\left(\frac{1}{\lambda_{1}^{\frac{\alpha}{2}}}+\frac{1}{\lambda_{2}^{\frac{\alpha}{2}}}+\frac{2}{\left(p_{a c t} \lambda_{R}\right)^{\frac{\alpha}{2}}}\right)\right],
$$

which is the closed-form solution of the end-to-end metric $p_{e x}$ for every value of $\alpha>2$. By inspecting Eq.(10), it can be easily shown that in low noise cases $(N \rightarrow 0)$ (interference limited environments) $p_{e x}$ becomes independent of the node intensities.

\section{B. Network lifetime}

At the end of a communication period $T_{m}$, the battery level of a relay, without taking EH into account, is described by

$$
L_{-e h}(m)=L_{I}-m t_{s}\left(2 P_{r}+P_{t} p_{a c t}\right)
$$

where $L_{I}$ is the initial energy level, $P_{r}$ is the power consumption at the reception mode, and $p_{\text {act }}$ is the probability of active relay, as described in Section III-A. In the case that the relay has EH capabilities, its battery level is described by

$$
L_{+e h}(m)=L_{I}-m t_{s}\left(2 P_{r}+P_{t} p_{a c t}\right)+m t_{s}\left(\sum_{i=1}^{3} \mathbb{E}\left\{P_{e h_{i}}\right\}\right),
$$

where $P_{e h_{i}}$ with $i \in 1,2,3$ are the instantaneous harvested powers at the corresponding time slots. The roots of Eq.(11) and Eq.(12) provide the relay's lifetime $m_{\max }$ for the two cases, respectively

$$
\begin{gathered}
m_{\text {max }_{-e h}}=\frac{L_{I}}{t_{s}\left(2 P_{r}+P_{t} p_{a c t}\right)} \text { and } \\
m_{\text {max }_{+e h}}=\frac{L_{I}}{\left[2 t_{s} P_{r}+t_{s} P_{t} p_{a c t}-t_{s} \sum_{i=1}^{3} \mathbb{E}\left\{P_{e h_{i}}\right\}\right]_{+}},
\end{gathered}
$$

where $[\xi]_{+}=\max (\xi, 0)$. In the case where $[\xi]_{+}=0$, the lifetime becomes infinite because the harvested energy is more

\footnotetext{
${ }^{1}$ Please note that, according to the Colouring theorem [13], the intensity of the relays in the third slot is $p_{a c t} \lambda_{R}$, since only the active relays transmit.
}

than the wasted. The instantaneous harvested power from EMR is the received power at the EHS from the interferers, scaled by the efficiency $\epsilon$ of the harvester, defined as

$$
P_{e h}=\epsilon \sum_{x \in \Phi} P_{t} h_{x} x^{-\alpha} .
$$

For the first two slots (i.e., $i=1,2$ ), the average harvested power $\mathbb{E}\left\{P_{e h_{i}}\right\}$ can be derived using Campbell's theorem for sums [7, 4.2]. Eq.(15) can then be written as

$$
\begin{aligned}
& \mathbb{E}\left\{P_{e h_{i}}\right\}=\epsilon P_{t} \mathbb{E}\left\{\sum_{x \in \Phi} h_{x} x^{-\alpha}\right\} \\
& \stackrel{(a)}{=} \epsilon P_{t} \mathbb{E}\left\{\sum_{x \in \Phi} x^{-\alpha}\right\} \stackrel{(b)}{=} \epsilon P_{t} \lambda_{i} \int_{\mathbb{R}^{2}} x^{-\alpha} \mathrm{d} x \\
& \stackrel{(a)}{=} \epsilon P_{t} \lambda_{i} \int_{0}^{2 \pi} \int_{\mathbb{E}_{d}\{d\}}^{\infty} r^{-\alpha} r \mathrm{~d} r \mathrm{~d} \phi \stackrel{(b)}{=} \frac{\epsilon P_{t} \lambda_{i} 2 \pi}{(\alpha-2)\left(2 \sqrt{\lambda_{i}}\right)^{2-\alpha}},
\end{aligned}
$$

where Eq.(17a) follows from the multiplicativity of the expected value ( $h_{x}$ and $x^{-\alpha}$ are independent), Eq.(17b) follows from the Campbell's theorem, Eq.(18a) follows from the integration in spherical coordinates and Eq.(18b) follows from the integration from the mean minimum distance, given in Eq.(8), to infinity. In the third slot, the relays are able to harvest energy from their own transmissions as well. Thus, Eq.(18) can be expressed as

$$
\mathbb{E}\left\{P_{e h_{3}}\right\}=\frac{\epsilon P_{t} p_{a c t} \lambda_{R} 2 \pi}{(\alpha-2)\left(2 \sqrt{p_{a c t} \lambda_{R}}\right)^{2-\alpha}}+\epsilon P_{t},
$$

where $\epsilon P_{t}$ is the additional part of self-harvested energy that is not affected by path loss and fading.

\section{Model VAlidation}

In this section, we validate the proposed theoretical framework via extensive simulations. We have developed a MATLAB simulator to create snapshots of the PPPs that exist on the plane, in order to measure the probability of successful data exchange and the lifetime gain in our system.

\section{A. Simulation setup}

In our experiments, we study the proposed system under high and low noise conditions (i.e., $N=10 \mathrm{dBm}$ and $N=$ $40 \mathrm{dBm})$. We use a path loss exponent $\alpha=3$, although it is possible to use any value $\alpha>2$. The mean value of the power fading coefficient is $\mu=1$ and the transmit power is $P_{t}=75 \mathrm{~mW}$, while the power for the reception mode is $P_{r}=$ $100 \mathrm{~mW}$. The conversion efficiency of the EHS is $\epsilon=0.1$ [11], while the intensities of the PPPs are $\lambda_{1}=\lambda_{2}=0.4$ and $\lambda_{R}=0.5$. Finally, the timeslot duration and the initial level of a relay's battery are $t_{s}=1 \mathrm{~s}$ and $L_{I}=1000 \mathrm{~J}$, respectively.

\section{B. Results}

Fig. 2(a) illustrates the lower bound of the probability of successful message exchange (Eq.(10)). In this figure, it can be seen that Eq.(6) is verified and that the lower bound is tight. The same remark is valid for other simulation parameters. Furthermore, as it was expected, the probability $p_{e x}$ is higher for low noise levels, in contrast to the case of high noise, where it reaches zero at lower thresholds. The probability $p_{e x}$ is decreasing as threshold increases, since less packets are decoded by the receiver. 


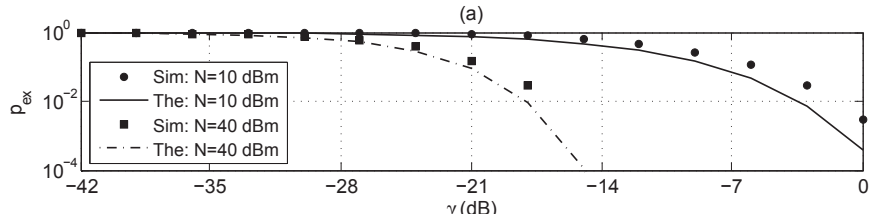

(b)
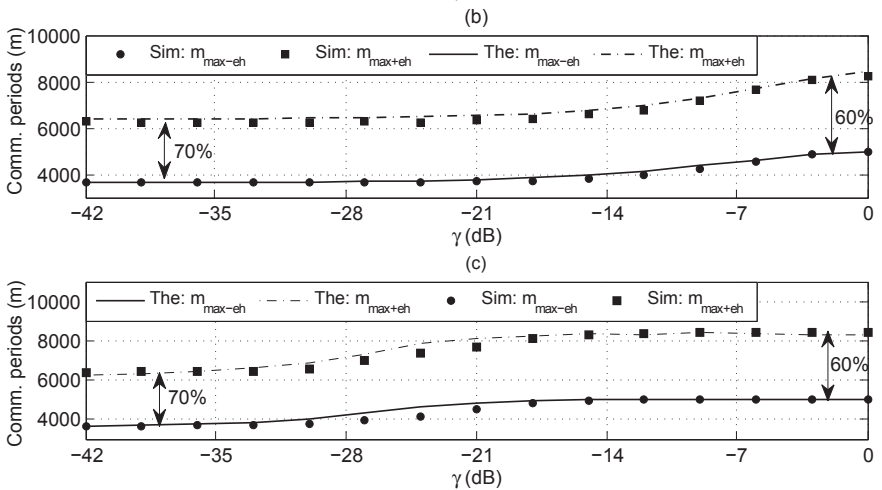

Fig. 2. (a) Probability of successful exchange vs. Threshold for different noise levels, (b) Lifetime vs. Threshold for low noise level $(N=10 \mathrm{dBm})$ and (c) Lifetime vs. Threshold for high noise level $(N=40 \mathrm{dBm})$.

Moreover, in Fig. 2(b), we present the lifetime of the network versus the threshold in low noise $(N=10 \mathrm{dBm})$, for networks without and with energy harvesting (Eq.(13) and Eq.(14), respectively). It is illustrated that the low bound is tight and that the benefits from harvesting EMR energy are significant, since the lifetime of the network can be increased up to $70 \%$ for low thresholds. We can observe from the figure that the lifetime reaches its maximum value at a threshold of around $0 \mathrm{~dB}$. This can be explained by noticing that the lower bound of $p_{e x}$ in Fig. 2(a) is significantly small at $0 \mathrm{~dB}$ for low noise environments, which means that the number of active relays is smaller and, thus, the node does not consume energy for transmission.

In Fig. 2(c), we plot the lifetime of the network versus the threshold for high noise $(N=40 \mathrm{dBm})$, and we observe the same behavior as in Fig. 2(b). However, in this case, the lifetime is reaching its maximum value at approximately $-21 \mathrm{~dB}$, which is the value of the threshold that $p_{e x}$ is almost zero for high noise environments (Fig. 2(a)).

In Fig. 3, we present an interesting result regarding the impact of the relay intensity $\lambda_{R}$ at the probability of successful exchange and the network lifetime. We can observe from Fig. 3(a) that $p_{e x}$ remains almost constant for low noise levels, despite the changes of the relay intensity. This result can be justified by the fact that the interference is higher for higher relay intensities, while at the same time the mean minimum distance from the transmitter (Eq.(8)) is also decreased. Thus, the SINR, as it is described in Eq.(1), is not affected by the differences in the relay intensity for negligible noise levels. On the other hand, as shown in Fig. 3(b), increasing the number of relays, increases the lifetime of the network for both low and high noise levels. Finally, it is evident that as the node intensity increases, the lifetime becomes infinite since the harvested energy is more than the consumed, as stated in Section III-B.

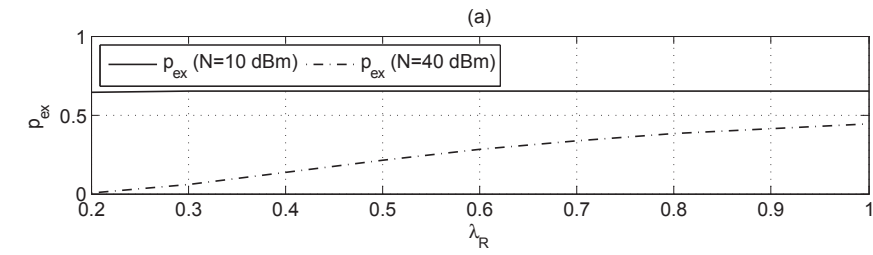

(b)

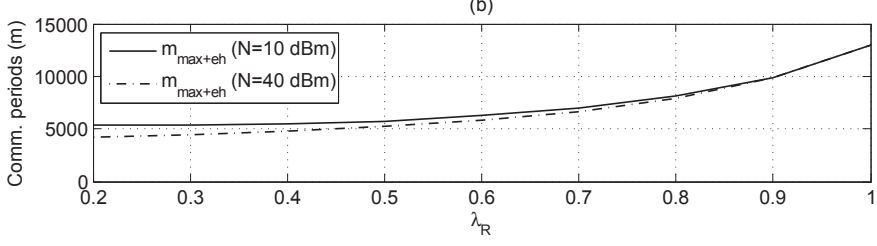

Fig. 3. (a) Probability of successful relay decoding vs. Relay intensity for high and low noise levels. $(\gamma=-30 \mathrm{~dB})$, and (b) Lifetime vs. Relay intensity for high and low noise levels.

\section{CONCLUSION}

In this paper, we provided a theoretical framework for studying the benefits of EMR energy harvesting in bidirectional network coded cooperative communications. It has been shown that the lifetime of the network can be increased up to $70 \%$. Moreover, it has been proved that, in low noise environments, increasing the relay intensity benefits the lifetime of the network, without compromising the QoS. In our future work, we plan to employ the proposed framework in constrained optimization problems for bidirectional cooperative networks.

\section{ACKNOWLEDGEMENTS}

This work has been funded by the research projects WSN4QoL (PIAP-GA-2011-286047), ESEE (324284), KINOPTIM (324491) and COPCAMS (332913).

\section{REFERENCES}

[1] R. Ahlswede et al., "Network information flow," IEEE Trans. Inf. Theory, vol. 46, no. 4, pp. 1204-1216, Jul. 2000.

[2] M. Xiao et al., "Design of network codes for multiple-user multiple relay wireless networks," IEEE Trans. Commun., vol. 60 , no. 12, pp. 37553766, Dec. 2012.

[3] M. Xiao and M. Skoglund, "Multiple-user cooperative communications based on linear network coding," IEEE Trans. Commun., vol. 58, no. 12, pp. 3345-3351, Dec. 2010.

[4] O. K. Rayel et al., "Energy Efficiency of Network Coded Cooperative Communications in Nakagami-m Fading," IEEE Signal Process. Lett., vol. 20, no. 10, pp. 960-963, Dec. 2010.

[5] Y. Chen and Q. Zhao, "On the lifetime of wireless sensor networks," IEEE Comm. Letters, vol. 9, no. 11, pp. 976-978, Nov. 2005.

[6] H. Jabbar et al., "RF energy harvesting system and circuits for charging of mobile devices," IEEE Trans. Consum. Electron., vol. 56, no. 1, pp. 247-253, Feb. 2010.

[7] M. Haenggi, Stochastic Geometry for Wireless Networks, Cambridge University Press, 1st ed., 2013.

[8] K. Huang, "Throughput of Wireless Networks Powered by Energy Harvesting", Proc. Asilomar Conf. Signals, Syst., Comput., Nov. 2011.

[9] W. Guo and S. Wang, "Radio-frequency energy harvesting potential: a stochastic analysis," Trans. Emerging Telecommun. Technol., vol. 24, no. 5, pp. 453-457, Aug. 2013.

[10] D. Wang et al., "Coverage and Lifetime Optimization of WSNs with Gaussian Distribution," IEEE Trans. Mobile Comput., vol. 7, no. 12, pp. 1444-1458, Dec. 2008

[11] S. Ladan et al., "Highly Efficient Compact Rectenna for Wireless Energy Harvesting Application," IEEE Microwave, vol. 14, no. 1, pp. 117-122, Feb. 2013.

[12] J. G. Andrews et al., "A Tractable Approach to Coverage and Rate in Cellular Networks," IEEE Trans. Commun., vol. 59, no. 11, pp. 31223134, Nov. 2011.

[13] J.F.C. Kingman, Poisson Processes, Oxford Univ. Press, 1st ed., 1993.

[14] M. R. Spiegel, Mathematical Handbook of Formulas and Tables, Schaum's Outline Series, McGraw-Hill, 1968. 